\title{
Recombinant Bacillus subtilis Spores Elicit Th1/Th17-Polarized Immune Response in a Murine Model of Helicobacter pylori Vaccination
}

\author{
Małgorzata Stasiłojéc ${ }^{1} \cdot$ Krzysztof Hinc $^{2}$ - Grażyna Peszyńska-Sularz ${ }^{3}$ • \\ Michał Obuchowski $^{2}$ - Adam Iwanicki ${ }^{2}$
}

Published online: 18 March 2015

(c) The Author(s) 2015. This article is published with open access at Springerlink.com

\begin{abstract}
Current progress in research on vaccines against Helicobacter pylori emphasizes the significance of eliciting the Th1/Th17-polarized immune response. Such polarization can be achieved by selection of appropriate antigen and adjuvant. In this study, we wanted to check the polarization of the immune response elicited by UreB protein of Helicobacter acinonychis delivered by recombinant Bacillus subtilis spores upon oral immunization. $B$. subtilis spores presenting fragment of UreB protein and able to express entire UreB in vegetative cells after germination were orally administered to mice along with aluminum hydroxide or recombinant spores presenting IL2 as an adjuvant. The pattern of cytokines secreted by sensitized splenocytes assessed by the cytometric bead array clearly indicated polarization of the immune response toward both Th1 and Th17 in mice immunized with the use of above-mentioned adjuvants. Obtained result is promising regarding the usage of recombinant spores in formulations of vaccines against $H$. pylori and line up with the current state of research emphasizing the key role of appropriate adjuvants.
\end{abstract}

Adam Iwanicki

adam.iwanicki@biotech.ug.edu.pl

1 Department of Medical Biotechnology, Intercollegiate Faculty of Biotechnology UG-MUG, University of Gdańsk, Dębinki 1, 80-211 Gdańsk, Poland

2 Department of Medical Biotechnology, Intercollegiate Faculty of Biotechnology UG-MUG, Medical University of Gdańsk, Gdańsk, Poland

3 Tri-City Animal Laboratory, Medical University of Gdańsk, Gdańsk, Poland
Keywords Bacillus subtilis $\cdot$ Spores $\cdot$ Helicobacter pylori $\cdot$ Oral vaccine $\cdot$ Th1 $\cdot$ Th17

\section{Introduction}

Helicobacter pylori, a Gram-negative microaerophilic bacterium, infects gastric mucosa of more than half of the world's population. It is a risk factor of such gastroduodenal diseases as peptic ulcers or gastric cancers $[1,2]$. Infection with $H$. pylori induces in gastric mucosa, a strong inflammatory response, characterized by infiltration of neutrophils and T- and B-cells. According to recent results, the major role in this response is assigned to T-helper cells of Th1 and Th17 types, which can be activated in the infected organism with consequent production of IFN- $\gamma$, IL17 , and TNF- $\alpha$ (reviewed in [3]). In spite of strong innate and adaptive immune responses elicited by $H$. pylori, infected patients usually fail to clear the infection without appropriate treatment. Current standards for therapy of $H$. pylori infections assume application of multi-drug regimens which can result in significant side effects. Antibiotics used in combination with such drugs as proton pump inhibitors lead to 80-90\% eradication; nevertheless, failures can contribute to development of antibiotic resistance and re-infection $[4,5]$.

Vaccinations appear to be an attractive alternative to the treatment with antibiotics. Most studies involve the use of urease subunits as antigens in vaccine formulations. Although currently some of such vaccines are used in clinical trials (phase I) [6-8], there is a common agreement that selection of appropriate bacterial antigens is crucial for development of an effective vaccine. One of the promising vaccine candidates is based on multi-epitope DNA vaccine with $\mathrm{CpG}$ oligonucleotides and heat-labile enterotoxin 
LTB as adjuvants [9]. In other trials, mice immunized with $H$. pylori opiA gene-encoded construct were able to develop protective immune response when co-administered with IL-2 gene-encoded construct and LTB [10]. In another example, the successful immunization was achieved with CagA, VacA, and UreB proteins of $H$. pylori in different arrangements along with an adjuvant leading to Th1 shift of cellular response [11]. All these examples clearly indicate the importance of appropriate adjuvants in formulations of a vaccine against infections with $H$. pylori. While most studies involved usage of strong immunogens (e.g., bacterial toxins), for this purpose, there is an increasing interest in application of immunomodulatory molecules as adjuvants, e.g., interleukins.

In our recent study, we have shown that recombinant Bacillus subtilis spores can also serve as antigen carriers in vaccines directed against $H$. pylori. In our approach, we have used $B$. subtilis spores presenting fragment of UreB protein of Helicobacter acinonychis and able to express entire UreB protein in vegetative cells upon germination. Such recombinant spores were able to elicit specific Th1biased cellular immune response when co-administered with IL-2 presenting spores serving as mucosal adjuvants [12]. B. subtilis spores seem to be very interesting platforms for antigen presentation and delivery in mucosal vaccines. Such properties as extreme resistance to harsh environmental conditions (e.g., stomach lumen) as well as the easiness of genetic manipulation make them very suitable for that purpose. This is also reflected in successful application of B. subtilis spore-based vaccines in development of protective immunity against such pathogens as Clostridium perfringens [13], Clostridium difficile [14], Clostridium tetani [15], or Rotavirus [16].

Here, we present results indicating that specific cellular immune response elicited in mice by recombinant $B$. subtilis spores delivering UreB of $H$. acinonychis does not only develop upon oral co-administration with IL-2-displaying spores but also used with aluminum hydroxide as adjuvant. Moreover, we show that in case of both formulations, this immune response is of Th1 and Th17 type.

\section{Methods}

\section{Ethics Statement}

This study was carried out in strict accordance with the recommendations in the institutional and national guidelines for animal care and use. The protocol was approved by the Committee on the Ethics of Animal Experiments of the Medical University of Gdańsk (Permit Number: 4/2010). All surgery was performed under isoflurane anesthesia, and all efforts were made to minimize suffering.

\section{Bacterial Strains}

Bacillus subtilis strains used in this study were 168 [17], BKH108 (UreB), and BKH121 (IL-2) [12]. BKH108 strain produces spores displaying fragment of UreB protein of $H$. acinonychis as fusion with spore coat protein $\operatorname{CotC}$. The $u r e B$ gene encoding entire UreB protein of $H$. acinonychis is expressed from $p_{r r n o}$ promoter. For detailed description, refer to [12].

\section{Preparation of Spores}

Sporulation was induced by the exhaustion method in DS (Difco-Sporulation) medium as described elsewhere [18]. After the final suspension in water, spores were treated at $65{ }^{\circ} \mathrm{C}$ for $1 \mathrm{~h}$ to kill any residual vegetative cells. The spore suspension was titrated immediately for $\mathrm{CFU} / \mathrm{ml}$ before freezing at $-22{ }^{\circ} \mathrm{C}$. By this method, we could reliably produce $6 \times 10^{10}$ spores per liter of DSM culture.

\section{Spore Germination}

Spores were heat activated at $80{ }^{\circ} \mathrm{C}$ for $20 \mathrm{~min}$. Next, serial dilutions were prepared, plated onto LB medium solidified with $1.5 \%$ agar, and incubated overnight at $37{ }^{\circ} \mathrm{C}$. Numbers of obtained colonies were used for calculation of germination efficiency.

\section{Immunizations}

Five groups of eight mice (female, BALB/c, 8 weeks) were immunized by oral route with suspensions of either spores expressing CotC-UreB3 (BKH108) with $3 \% \mathrm{Al}(\mathrm{OH})_{3}$, both CotC-UreB3 (BKH108) and CotB-linker-IL-2 (BKH121) (1:1) or control spores (wild-type strain 168). A naive, non-immunized control group was included. Oral immunizations were performed with $1.0 \times 10^{10}$ spores in a volume of $0.2 \mathrm{ml}$ of water administered by intragastric lavage on days $1,3,5,22,24,26,43,45$, and 47. Animals were sacrificed on day 61 , and serum samples and spleens were collected.

\section{Indirect ELISA for Detection of Antigen-Specific Serum}

UreB-specific antibodies in saponin extracts of gastrointestinal tracts or sera of immunized animals were detected as previously described [12]. Briefly, plates were coated with $100 \mu \mathrm{l}$ per well of the specific antigen $(2 \mu \mathrm{g} / \mathrm{ml}$ in carbonate/bicarbonate buffer) and left at room temperature overnight. Antigen was UreB-purified protein. After blocking with $0.5 \% \mathrm{BSA}$ in PBS for $1 \mathrm{~h}$ at $37{ }^{\circ} \mathrm{C}$, samples were applied using a two-fold dilution series starting with a 
$1 / 20$ dilution in ELISA diluent buffer (0.1 M Tris- $\mathrm{HCl}, \mathrm{pH}$ 7.4; $3 \%(\mathrm{w} / \mathrm{v}) \mathrm{NaCl} ; 0.5 \%(\mathrm{w} / \mathrm{v}) \mathrm{BSA} ; 10 \%(\mathrm{v} / \mathrm{v})$ sheep serum (Sigma); $0.1 \%$ (v/v) Triton-X-100; $0.05 \%$ (v/v) Tween-20). Every plate carried replicate wells of a negative control (a 1/20 diluted pre-immune serum) and a positive control (serum from mice immunized intraperitoneally with UreB-purified protein). Plates were incubated for $2 \mathrm{~h}$ at $37^{\circ} \mathrm{C}$ before addition of anti-mouse AP conjugates (Sigma). Plates were incubated for a further $1 \mathrm{~h}$ at $37{ }^{\circ} \mathrm{C}$ then developed using the substrate pNPP (paraNitrophenylphosphate; Sigma). Reactions were stopped using $2 \mathrm{M} \mathrm{H}_{2} \mathrm{SO}_{4}$.

\section{Isolation of Splenocytes}

Mice were sacrificed, and spleens were isolated as previously described [12]. Briefly, mice were sacrificed, and spleens were aseptically removed. The spleens were then perfused with RPMI-1640 (supplemented with $10 \%$ heatinactivated fetal calf serum, $25 \mathrm{mM}$ HEPES, $2 \mathrm{mM}$ L-glutamine, $1 \mathrm{mM}$ sodium pyruvate, $100 \mathrm{IU} / \mathrm{ml}$ penicillin, and $100 \mathrm{mg} / \mathrm{ml}$ streptomycin) using 5-ml syringe fitted with $26 \mathrm{G}$ needle to obtain single-cell suspension of splenocytes. The splenocytes suspension was then centrifuged at $300 \times g$ for $15 \mathrm{~min}$. The RBCs were lysed by hypotonic shock using $3 \mathrm{ml}$ of $0.84 \%$ of sterile $\mathrm{NH}_{4} \mathrm{Cl}$ or ACK lysis buffer for $5 \mathrm{~min}$. The cells were then washed thrice with RPMI-1640 to remove lysed $\mathrm{RBCs}$ and $\mathrm{NH}_{4} \mathrm{Cl}$.

\section{Activation of Splenocytes}

Splenocytes $\left(2 \times 10^{5} / \mathrm{mL}\right)$ were cultured in the presence or absence of UreB antigen for $48 \mathrm{~h}$. Samples of supernatants containing released cytokines were collected and stored at $-80{ }^{\circ} \mathrm{C}$

\section{Flow Cytometry}

Levels of IL-10, IL-17, TNF, IFN- $\gamma$ IL-6, IL-4, and IL-2 secreted by sensitized cells were determined by cytometric bead array (CBA) Mouse Th1/Th2/Th17 Cytokine Kit (BD) kit according to manufacturer's protocol. Measurements were performed using Accuri C6 Flow Cytometer (BD) and results processed with system software. Six technical repeats were done for each animal in the group. Results were statistically evaluated using Student's $t$ test.

\section{Results}

IL-2-presenting spores were shown to have mucosal adjuvant properties [12]. We were interested, whether classical adjuvant, aluminum hydroxide, would also help in eliciting a specific immune response in Balb/c mice upon oral administering of spores of BKH108 (UreB) recombinant B. subtilis strain. Our previous studies have shown that efficient immune response to recombinant spores was strictly dependent on the use of an adjuvant. In the current study, we performed immunization cycle using spores of BKH108 strain along with aluminum hydroxide. As a reference in immunizations, we used spores of BKH108 strain mixed with IL-2-presenting spores (BKH121). Prior to immunizations, we tested the influence of aluminum hydroxide on germination of spores of the wild-type and BKH108 strains. We observed lowered germination efficiency of spores incubated in suspension of this compound (Table 1). Animals immunized with both formulations showed no side effects or weight loss associated with immunizations (data not shown).

Upon completion of immunization cycle, we performed characterization of developed immune response. We observed very low levels of UreB-specific IgG antibodies in sera of immunized animals (Fig. 1) and no such IgG or IgA antibodies in saponin extracts of isolated gastrointestinal tracts (data not shown). Systemic antigen-specific cellular immune response was assessed using the CBA performed on sensitized splenocytes isolated from immunized animals. The composition of the CBA assays used in the research enabled characterizing the type (Th1, Th2, or Th17) of observed immune response as Th1- and Th17-polarized (Fig. 2). In case of both formulations, profiles of secreted cytokines were very similar, but higher amounts of cytokines were observed in samples prepared with splenocytes of animals immunized with BKH108 spores and aluminum hydroxide as adjuvant. Cytokines characteristic for Th1 response: IL-2, IFN- $\gamma$, and TNF- $\alpha$ were present in culture supernatants at significantly higher concentrations in case of sensitized splenocytes as compared to control splenocytes (naïve mice or mice immunized with 168 carrier spores). Similarly, levels of IL-17 and IL-6 were significantly higher in samples of supernatants of sensitized cells. At the same time, levels of cytokines characteristic

Table 1 Efficiency of germination of spores produced by the wildtype 168 and BKH108 strains

\begin{tabular}{lcc}
\hline & 168 & \multicolumn{1}{c}{ BKH108 } \\
\hline $\mathrm{H}_{2} \mathrm{O}$ & $100 \pm 10^{\mathrm{a}}$ & $100 \pm 13.1^{\mathrm{a}}$ \\
$\mathrm{Al}(\mathrm{OH})_{3}$ & $47.9 \pm 5.1$ & $17.98 \pm 7.9$
\end{tabular}

${ }^{\text {a }}$ Germination of spores suspended in water or $3 \%$ aluminum hydroxide was analyzed as described in "Methods" section. Numbers represent percentages of germinated spores along with standard deviations. Amounts of germinated spores incubated in water were taken as 100 . The presented results are averages of three independent experiments 


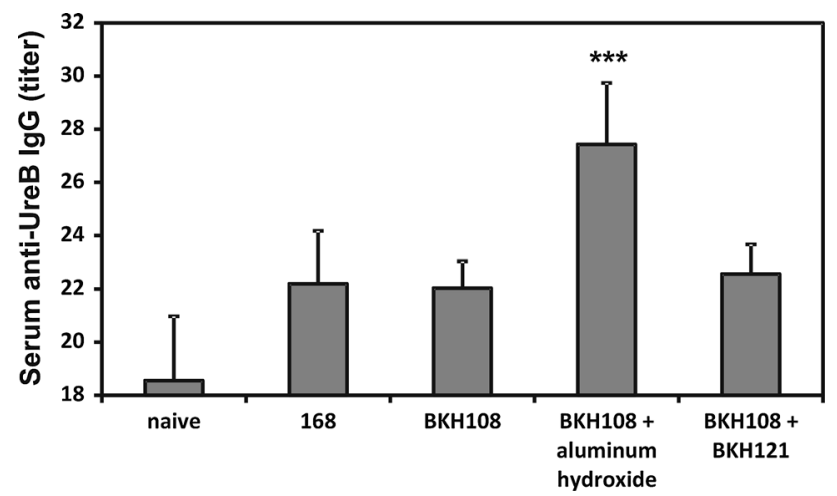

Fig. 1 Endpoint titration of IgG antibody to UreB in sera collected from mice upon completion of immunization cycle (day 61). Mice were orally immunized with wild-type 168 or recombinant BKH108 (UreB) and BKH121 (IL-2) spores as described in "Methods" section. Each bar represents average of UreB-specific IgG titers in sera of eight animals. Error bars represent standard deviation. Statistical significance versus 168 assessed by Student's $t$ test $* * * p<0.001$

for Th2 response: IL-4, and IL-10, were lowered as compared to control cells.

\section{Discussion}

The development of an efficient vaccine against $H$. pylori infections seems to be a real challenge. In spite of increasing number of studies aimed on creation of such vaccine, still no satisfactory solution has been found. Three critical issues regarding the development of an efficient anti- $H$. pylori vaccine have been proposed, and these are selection of appropriate bacterial antigens, safe and effective adjuvants, and a route of delivery [3].

UreB subunit of $H$. pylori urease has already been used in several studies as an antigen in immunizations against this pathogen. While most of the researches have been done using such recombinant bacteria as Salmonella typhimurium [19] or Lactococcus lactis [20], few works involved the use of recombinant plants (rice [21], carrot [22], or peanuts [23]) expressing UreB protein. Regarding the route of immunization, oral vaccines seem to be the most interesting since they should lead to the delivery of an antigen directly to the site of infection. Moreover, the easiness of administering of such vaccines is an additional benefit of such vaccines. Due to these facts $B$. subtilis spores seem to be a very good platform for antigen delivery. Indeed, they have already been applied for construction of promising vaccine candidates [13-16].

We wanted to further investigate usefulness of recombinant $B$. subtilis BKH108 spores which deliver the UreB protein of $H$. acinonychis, a close relative of $H$. pylori, as antigen. We decided to use urease subunit $\mathrm{B}$ from
$H$. acinonychis to avoid any potential patent issues, which may arise in case of use of UreB from $H$. pylori. As the recent progress in the research on vaccines against $H$. pylori suggested the need of application of an appropriate adjuvant, we decided to use for this purpose as a wellknown compound, aluminum hydroxide. Aluminum compounds have been used for decades as efficient adjuvants in subcutaneous vaccines, leading to the induction of Th2polarized immune response [24-26]. $\mathrm{Al}(\mathrm{OH})_{3}$ has been shown to induce local decrease in expression of the Th2 cytokine IL-10 and local increase of expression of the Th1 cytokine IL-12 in the stomach in parenteral immunizations [27]. This compound has also been used as adjuvant in mucosal immunizations by intranasal [28, 29] and oral [30] routes. In our previous study, we were able to elicit UreBspecific immune response in mice orally immunized with BKH108 spores only upon co-administering with IL-2 presenting spores [12]. Since IL-2 is a strong immunomodulatory cytokine, it would require very detailed optimization in composing of safe and efficient vaccine formulation consisting of these two types of spores. The decision to use aluminum hydroxide as adjuvant in current study was directed to overcome such issues. Our idea proved to be right, since we obtained efficient UreBspecific immune response in immunized animals. We went further and tried to characterize the polarization of elicited immune response. As shown in Fig. 2, the profile of secreted cytokines suggests the response to be both Th1- and Th17-polarized, with simultaneous inhibition of Th2 polarization. This observation is supported by results of titration of UreB-specific antibodies in the sera of immunized animals. Although we noticed some minimal titers of these antibodies (Fig. 1), we consider them as negligible. It is important to notice that the same pattern of cytokines production was observed for splenocytes isolated from mice immunized orally with BKH108 spores and BKH121 spore, presenting IL-2 (Fig. 2). Obtained polarization of the immune response is promising in light of recent studies on responses of the immune system to infection with $H$. pylori. As reviewed by D'Elios et al. [3], infection with this bacterium leads to activation of both Th1 and Th17 cells with subsequent production of IFN- $\gamma$, IL-17, and TNF- $\alpha$. Moreover, it has been shown that vaccinations promoting Th1 or Th17 immunity are sufficient to protect mice from $H$. pylori $[31,32]$.

Interestingly, B. subtilis spores presenting UreB of H. pylori have recently been shown by Zhou et al. to be efficient in protecting of orally immunized mice against infection with this bacterium [33]. This observation does not line up with our previous [12] and current results, since we observed no immune response elicited by BKH108 spores administered without any adjuvant. These discrepancies might partially be explained with differences of 

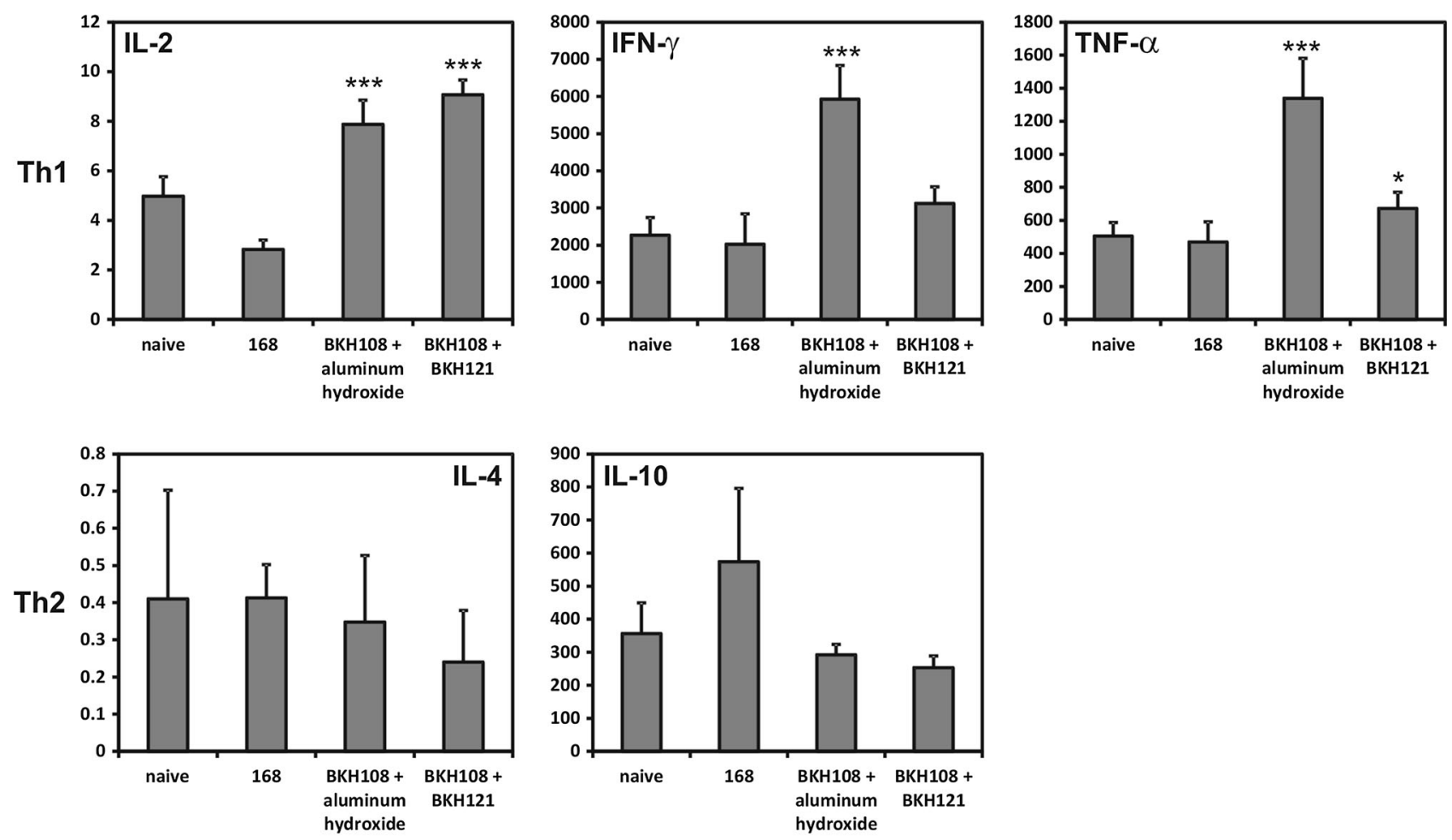
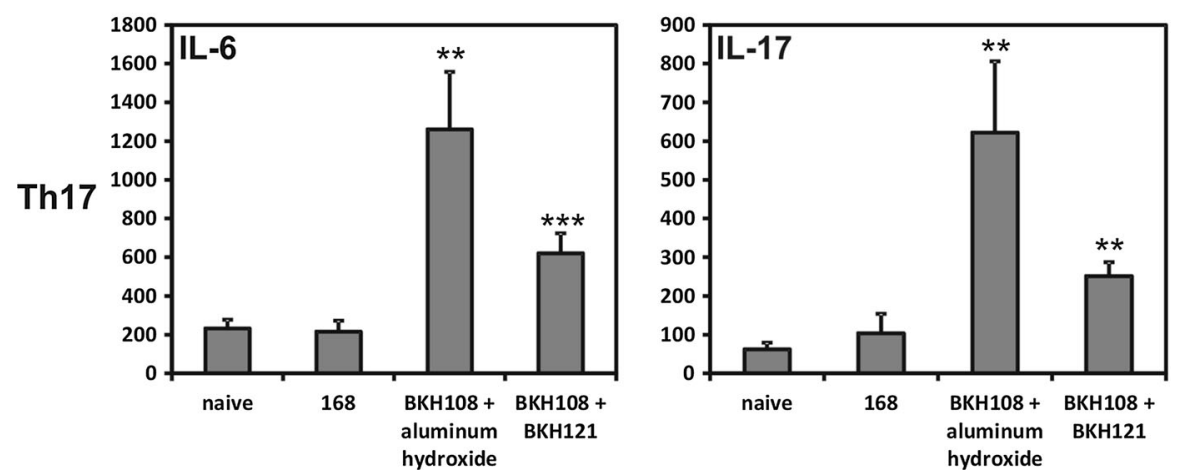

Fig. 2 Polarization of immune response is elicited by recombinant spores as assessed by analysis of profiles of cytokines secreted by sensitized splenocytes. Splenocytes were isolated from mice orally immunized with the wild-type 168 or recombinant BKH108 (UreB) and BKH121 (IL-2) spores as described in "Methods" section. Levels of cytokines $(\mathrm{pg} / \mathrm{ml})$ indicated in each graph were measures by flow cytometry using the cytometric bead array. Graphs are arranged in

UreB used as antigen displayed on the surface of $B$. subtilis spores. While Zhou et al. reported usage of $H$. pylori UreB [33], in our experiments, we used UreB of $H$. acinonychis. This is surprising, up to some extent, because UreB proteins from both microorganisms share $99 \%$ identity of amino acid sequence. Moreover, $H$. acinonychis is an animal pathogen closely related to $H$. pylori and recognized as a useful in vivo model to study $H$. pylori virulence mechanisms [34]. The same study reports production of UreB-specific antibodies in immunized animals [33], but due to the specific way of data presentation $\left(\mathrm{OD}_{450}\right.$ instead groups of cytokines characteristic for polarizations of the immune response indicated with Th1, Th2, or Th17. The presented results are averages of cytokines levels secreted by splenocytes isolated from groups of eight animals. Error bars indicate standard deviation. Statistical significance versus 168 assessed by Student's $t$ test $* p<0.05, * * p<0.01, * * * p<0.001$

of antibody titer), it was not possible to compare it with results obtained in our study. It is also worth mentioning that our results are in agreement with previous studies, in which $B$. subtilis spores presenting antigens elicited strong Th1-biased immune response with no (or very low) humoral response [35, 36].

To conclude, we have shown that usage of aluminum hydroxide in oral immunizations with recombinant spores helped elicit the immune response specific for an antigen presented on the spore surface. Moreover, the polarization of observed response to UreB protein goes along with the 
current research on vaccines against $H$. pylori suggesting, that formulations basing on recombinant B. subtilis spores, supplemented with an appropriate adjuvant, may be useful in protection against infection with this pathogen.

Acknowledgments This research was supported by the Polish National Science Centre Grant No. N401 279439 and by the Foundation for Polish Science Grant HOMING PLUS/2010-2/3.

Open Access This article is distributed under the terms of the Creative Commons Attribution License which permits any use, distribution, and reproduction in any medium, provided the original author(s) and the source are credited.

\section{References}

1. NIH Consensus Conference. (1994). Helicobacter pylori in peptic ulcer disease. Journal of the American Medical Association, 272, 1887-1897.

2. World Health Organization. (1994). Infection with Helicobacter pylori. Schistosomes, liver flukes and Helicobacter pylori (pp. 177-241). Lyon: International Agency for Research on Cancer.

3. D’Elios, M. M., \& Czinn, S. J. (2014). Immunity, inflammation and vaccines for Helicobacter pylori. Helicobacter, 19(suppl 1), S19-S26.

4. Malfertheiner, P., Megraud, F., O'Morain, C. A., Atherton, J., Axon, A. T., et al. (2012). Management of Helicobacter pylori infection-the Maastricht IV/florence consensus report. Gut, 61, 646-664.

5. Tepes, B., O’Connor, A., Gisbert, J. P., \& O’Morain, C. (2012). Treatment of Helicobacter pylori infection 2012. Helicobacter, 17(suppl 1), S36-S42.

6. Bumann, D., Metzger, W. G., Mansouri, E., Palme, O., Wendland, M., et al. (2001). Safety and immunogenicity of live recombinant Salmonella enterica serovar Typhi Ty21a expressing urease A and B from Helicobacter pylori in human volunteers. Vaccine, 20, 845-852.

7. DiPetrillo, M. D., Tibbetts, T., Kleanthous, H., Killeen, K. P., \& Hohmann, E. L. (1999). Safety and immunogenicity of phoP/ phoQ-deleted Salmonella typhi expressing Helicobacter pylori urease in adult volunteers. Vaccine, 18, 449-459.

8. Rizos, K., Lattemann, C. T., Bumann, D., Meyer, T. F., \& Aebischer, T. (2003). Autodisplay: efficacious surface exposure of antigenic UreA fragments from Helicobacter pylori in Salmonella vaccine strains. Infection and Immunity, 71, 6320-6328.

9. Moss, S. F., Moise, L., Lee, D. S., Kim, W., Zhang, S., et al. (2011). HelicoVax: epitope-based therapeutic Helicobacter pylori vaccination in a mouse model. Vaccine, 29, 2085-2091.

10. Chen, J., Lin, L., Li, N., \& She, F. (2012). Enhancement of Helicobacter pylori outer inflammatory protein DNA vaccine efficacy by co-delivery of interleukin-2 and B subunit heat-labile toxin gene encoded plasmids. Microbiology and Immunology, 56, 85-92.

11. Liu, K. Y., Shi, Y., Luo, P., Yu, S., Chen, L., et al. (2011). Therapeutic efficacy of oral immunization with attenuated Salmonella typhimurium expressing Helicobacter pylori CagA, VacA and UreB fusion proteins in mice model. Vaccine, 29, 6679-6685.

12. Hinc, K., Stasiłojć, M., Piątek, I., Peszyńska-Sularz, G., Isticato, R., et al. (2014). Mucosal adjuvant activity of IL-2 presenting spores of Bacillus subtilis in a murine model of Helicobacter pylori vaccination. PLoS One, 9, e95187.
13. Hoang, T. H., Hong, H. A., Clark, G. C., Titball, R. W., \& Cutting, S. M. (2008). Recombinant Bacillus subtilis expressing the Clostridium perfringens alpha toxoid is a candidate orally delivered vaccine against necrotic enteritis. Infection and Immunity, 76, 5257-5265.

14. Permpoonpattana, P., Hong, H. A., Phetcharaburanin, J., Huang, J. M., Cook, J., et al. (2011). Immunization with Bacillus spores expressing toxin A peptide repeats protects against infection with Clostridium difficile strains producing toxins A and B. Infection and Immunity, 79, 2295-2302.

15. Duc, L. H., Hong, H. A., Fairweather, N., Ricca, E., \& Cutting, S. M. (2003). Bacterial spores as vaccine vehicles. Infection and Immunity, 71, 2810-2818.

16. Lee, S., Belitsky, B. R., Brinker, J. P., Kerstein, K. O., Brown, D. W., et al. (2010). Development of a Bacillus subtilis-based rotavirus vaccine. Clinical and Vaccine Immunology, 7, $1647-1655$

17. Anagnostopoulos, C., \& Crawford, I. P. (1961). Transformation studies on the linkage of markers in the tryptophan pathway in Bacillus subtilis. Proceedings of the National Academy of Sciences of the United States of America, 47, 378-390.

18. Nicholson, W. L., \& Setlow, P. (1990). Sporulation, germination and outgrowth. In C. Harwood \& S. Cutting (Eds.), Molecular biological methods for Bacillus (pp. 391-450). Chichester, UK: Wiley.

19. Gómez-Duarte, O. G., Lucas, B., Yan, Z. X., Panthel, K., Haas, R., et al. (1998). Protection of mice against gastric colonization by Helicobacter pylori by single oral dose immunization with attenuated Salmonella typhimurium producing urease subunits A and B. Vaccine, 16, 460-471.

20. Lee, M. H., Roussel, Y., Wilks, M., \& Tabaqchali, S. (2001). Expression of Helicobacter pylori urease subunit B gene in Lactococcus lactis MG1363 and its use as a vaccine delivery system against $H$. pylori infection in mice. Vaccine, 19, 3927-3935.

21. Gu, Q., Han, N., Liu, J., \& Zhu, M. (2006). Expression of Helicobacter pylori urease subunit B gene in transgenic rice. Biotechnology Letters, 28, 1661-1666.

22. Zhang, H., Liu, M., Li, Y., Zhao, Y., He, H., et al. (2010). Oral immunogenicity and protective efficacy in mice of a carrotderived vaccine candidate expressing UreB subunit against Helicobacter pylori. Protein Expression and Purification, 69, 127-131.

23. Yang, C. Y., Chen, S. Y., \& Duan, G. C. (2011). Transgenic peanut (Arachis hypogaea L.) expressing the urease subunit B gene of Helicobacter pylori. Current Microbiology, 63, 387-391.

24. Brewer, J. M., Conacher, M., Hunter, C. A., Mohrs, M., Brombacher, F., \& Alexander, J. (1999). Aluminium hydroxide adjuvant initiates strong antigenspecific Th2 responses in the absence of IL-4- or IL-13-mediated signaling. Journal of Immunology, 163, 6448-6454.

25. Brewer, J. M., Conacher, M., Satoskar, A., Bluethmann, H., \& Alexander, J. (1996). In interleukin-4-deficient mice, alum not only generates $\mathrm{T}$ helper 1 responses equivalent to Freund's complete adjuvant, but continues to induce $\mathrm{T}$ helper 2 cytokine production. European Journal of Immunology, 26, 2062-2066.

26. Grun, J. L., \& Maurer, P. H. (1989). Different T helper cell subsets elicited in mice utilizing two different adjuvant vehicles: the role of endogenous IL-1 in proliferative responses. Cellular Immunology, 121, 134-145.

27. Minoura, T., Kato, S., Otsu, S., Fujioka, T., Iinuma, K., \& Nishizono, A. (2003). Childhood Helicobacter pylori infection in a murine model: maternal transmission and eradication by systemic immunization using bacterial antigen-aluminium hydroxide. Clinical and Experimental Immunology, 134, 32-37. 
28. Isaka, M., Yasuda, Y., Kozuka, S., Miura, Y., Taniguchi, T., et al. (1998). Systemic and mucosal immune responses of mice to aluminium-adsorbed or aluminium-non-adsorbed tetanus toxoid administered intranasally with recombinant cholera toxin B subunit. Vaccine, 16, 1620-1626.

29. Isaka, M., Yasuda, Y., Kozuka, S., Taniguchi, T., Miura, Y., et al. (1999). Intranasal or subcutaneous co-administration of recombinant cholera toxin B subunit stimulates only a slight or no level of the specific IgE response in mice to tetanus toxoid. Vaccine, 17, 944-948.

30. Eastcott, J. W., Holmberg, C. J., Dewhirst, F. E., Esh, T. R., Smith, D. J., \& Taubman, M. A. (2001). Oligonucleotide containing $\mathrm{CpG}$ motifs enhances immune response to mucosally or systemically administered tetanus toxoid. Vaccine, 19, 1636-1642.

31. Ding, H., Nedrud, J. G., Blanchard, T. G., Zagorski, B. M., Li, G., Shiu, J., et al. (2013). Th1-mediated immunity against Helicobacter pylori can compensate for lack of Th17 cells and can protect mice in the absence of immunization. PLoS One, 8, e69384.

32. Velin, D., Favre, L., Bernasconi, E., Bachmann, D., Pythoud, C., Saiji, E., et al. (2009). Interleukin-17 is a critical mediator of vaccine-induced reduction of Helicobacter infection in the mouse model. Gastroenterology, 136, 2237-2246.

33. Zhou, Z., Gong, S., Li, X., Yang, Y., Guan, R., Zhou, S., et al. (2014). Expression of Helicobacter pylori urease B on the surface of Bacillus subtilis spores. Journal of Medical Microbiology,. doi:10.1099/jmm.0.076430-0.

34. Dailidiene, D., Dailide, G., Ogura, K., Zhang, M., Mukhopadhyay, A. K., Eaton, K. A., et al. (2014). Helicobacter acinonychis: genetic and rodent infection studies of a Helicobacter pylori-like gastric pathogen of cheetahs and other big cats. Journal of Bacteriology, 186, 356-365.

35. Mauriello, E. M., Cangiano, G., Maurano, F., Saggese, V., De Felice, M., Rossi, M., et al. (2007). Germination-independent induction of cellular immune response by Bacillus subtilis spores displaying the $\mathrm{C}$ fragment of the tetanus toxin. Vaccine, 25, 788-793.

36. Isticato, R., Sirec, T., Treppiccione, L., Maurano, F., De Felice, M., Rossi, M., et al. (2013). Non-recombinant display of the B subunit of the heat labile toxin of Escherichia coli on wild type and mutant spores of Bacillus subtilis. Microbial Cell Factories, 12, 98. doi:10.1186/1475-2859-12-98. 